\title{
THE FLUCTUATION INDUCED PSEUDOGAP IN THE INFRARED OPTICAL CONDUCTIVITY OF HIGH TEMPERATURE SUPERCONDUCTORS
}

\author{
Francesca Federici and Andrei A. Varlamovi \\ Laboratorio "Forum" dell'Istituto Nazionale di Fisica della Materia, \\ Dipartimento di Fisica, Università di Firenze, \\ Largo E.Fermi, 2, 50125 Firenze, Italy
}

(October 29, 2018)

\begin{abstract}
We study the effect of fluctuations on the ac conductivity of a layered superconductor both for $c$-axis and $a b$-plane electromagnetic wave polarizations. The fluctuation contributions of different physical nature and signs (paraconductivity, Maki-Thompson anomalous contribution, one-electron density of states renormalization) are found to be suppressed by the external field at different characterisitic frequencies $\left(\omega_{\mathrm{AL}} \sim T-T_{\mathrm{c}}, \omega_{\mathrm{MT}} \sim \max \left\{T-T_{\mathrm{c}}, \tau_{\varphi}^{-1}\right\}, \omega_{\mathrm{DOS}} \sim \min \left\{T, \tau^{-1}\right\}\right.$ for the $2 D$ case). As a result the appearance of the nonmonotonic frequency dependence (pseudogap) in the infrared optical conductivity of HTS film is predicted. The effect has to be especially pronounced in the case of the electromagnetic field polarization along $c$-axis.
\end{abstract}

\section{INTRODUCTION}

Recently it was demonstratede H $^{-1}$ that due to such specific properties of HTS as the extremely strong anisotropy and the small coherence length the fluctuation renormalization of the one-electron density of states (or, in other words, the opening of the fluctuation pseudogap above $T_{\mathrm{c}}$ ) 3 can play an important role in the explanation of their normal phase properties near the superconducting transition. The depression of the one-electron density of states (DOS) on the Fermi level results in the decrease of related thermodynamic and transport properties which are proportional to that one. It is important that this effect has an opposite sign with respect to the traditionally discussed Aslamazov-Larkin (AL) and Maki-Thompson (MT) corrections though it remains less singular in $T-T_{\mathrm{c}} \mathrm{E}$.

The most explicit manifestation of the effect discussed can be observed in the $c$-axis transport properties of layered materials above $T_{\mathrm{c}}$, such as the fluctuation conductivity and the magnetoresistance, where paraconductivity and $\mathrm{Maki}_{\mathrm{a}}$ Thompson contribution in the $2 D$ regime turn out to be suppressed by the square of the interlayer trasmittance 01002. Another interesting example of the evident appearance of the negative DOS contribution is the case of the NMR relaxation rate for which the AL-like contribution is rigorously forbidden for the singlet pairing and the MT contribution compets with the DOS ond 13 15. Strong pairbreaking weaks the former and the latter leads to the decrease of the NMR relaxation rate at the edge of the transition 16 .

In the present communication we attract the attention to another possibility to suppress the main AL and MT contributions by means of an high frequency electromagnetic field applied. Basing on the Drude origin of the normal part of the optical conductivity one can expect that noticeable changes in the electromagnetic wave reflectivity versus frequency take place for $\omega \sim \tau^{-1}$. But it turns out that the frequency dependence of paraconductivityand MakiThompson anomalous contribution in the vicinity of $T_{\mathrm{c}}$ appears long before, for $\omega \sim \min \left\{T-T_{\mathrm{c}}, \tau_{\varphi}^{-1}\right\} 11$. 18 . These positive contributions compet with the negative $\sigma^{\mathrm{DOS}}(\omega)$ which, as we will see below, weakly depends on frequency (only in the scale $\omega \sim \min \left\{T, \tau^{-1}\right\}$ ). This competition results in the rapid decay of the dissipative processes already at frequencies of the order of $\omega \sim T-T_{\mathrm{c}}$ and in the appearance of the transparency window up to $\omega \sim \min \left\{T, \tau^{-1}\right\}$. The following high frequency behavior of $\operatorname{Re}[\sigma(\omega)]$ is mostly governed by $\sigma^{\mathrm{n}}(\omega)$ which decreases, in agreement with Drude law, for $\omega \gtrsim \tau^{-1}$.

Below we will study the ac fluctuation conductivity tensor for a layered superconductor taking into account all contributions and paying attention to the most interesting case of $c$-axis polarization of the field. Nevertheless, for completness, the $a b$-plane results of the old paper of Aslamazov and Varlamov17 will be re-examined and discussed in application to the novel HTS layered systems.

\section{THE MODEL AND SOME DEFINITIONS}

We study the optical conductivity of layered superconductors which can be expressed by the retarded analitical continuation of the current-current correlator (electromagnetic response operator) $Q^{(R)}(\omega)$ :

$$
\operatorname{Re}\left[\sigma_{\alpha \beta}(\omega)\right]=-\frac{\operatorname{Im}\left[Q_{\alpha \beta}^{(R)}(\omega)\right]}{\omega}
$$


The problem of fluctuation conductivity of layered superconductors has been extensively studied and for the detailed description of the model and the diagrammatic presentation of the electromagnetic response operator tensor $Q_{\alpha \beta}$ we refer to 11. So below we will list only the necessary definitions and results following the notations of that article.

We assume the electron spectrum of the layered metal in the form

$$
\xi(\mathbf{p})=\epsilon_{0}(\mathbf{p})+J \cos \left(p_{z} s\right)-E_{\mathrm{F}},
$$

where $\epsilon_{0}(\mathbf{p})=\mathbf{p}^{2} /(2 m), p \equiv\left(\mathbf{p}, p_{z}\right), \mathbf{p} \equiv\left(p_{x}, p_{y}\right)$ is a two-dimensional, intralayer wavevector, and $J$ is an effective quasiparticle nearest-neighbour interlayer hopping energy. The Fermi surface defined by the condition $\xi\left(p_{\mathrm{F}}\right)=0$ is a corrugated cylinder, and $E_{\mathrm{F}}$ is the Fermi energy. This model spectrum is most appropriate for highly anisotropic layered materials, for which $J / E_{\mathrm{F}} \ll 1$.

As it is well established now, the electron mean free path in HTS layered single crystals or epitaxial films turns out to be of the same order or several times larger than the coherence length $\xi_{a b}(0)$, so the parameter $T_{\mathrm{c}} \tau \sim 1$ and the theory has to be constructed for an arbitrary impurity concentration.

Intralayer quasiparticle scattering is included in the single quasiparticle normal state Green's function by means of the relaxation time $\tau$ :

$$
G\left(\mathbf{p}, \omega_{n}\right)=\frac{1}{i \tilde{\omega}_{n}-\xi(\mathbf{p})}
$$

where $\tilde{\omega}_{n}=\omega_{n}\left[1+\frac{1}{2} /\left(2\left|\omega_{n}\right| \tau\right)\right]$.

The "Cooperon"19 (triangle vertex accounting the interference in the impurity avaraging of the pairs of Green's functions), which is necessary in the following calculations, may be expressed, for an arbitrary impurity concentration, 17,11 as:

$$
C\left(\mathbf{q}, \omega_{n}, \omega_{n^{\prime}}\right)=\left[1-\frac{\Theta\left(-\omega_{n} \omega_{n^{\prime}}\right)}{\tau\left(\tilde{\omega}_{n}-\tilde{\omega}_{n^{\prime}}\right)}\left(1-\frac{\left\langle[\xi(\mathbf{p})-\xi(\mathbf{q}-\mathbf{p})]^{2}\right\rangle}{\left(\tilde{\omega}_{n}-\tilde{\omega}_{n^{\prime}}\right)^{2}}\right)\right]^{-1}
$$

where $\Theta(x)$ is the Heaviside step function, and $\langle\cdots\rangle$ denotes an average over the Fermi surface. Performing the Fermi surface average with the spectrum (2), we find:

$$
\left\langle[\xi(\mathbf{p})-\xi(\mathbf{q}-\mathbf{p})]^{2}\right\rangle=\frac{1}{2}\left(v_{\mathrm{F}}^{2} \mathbf{q}^{2}+4 J^{2} \sin ^{2}\left(q_{z} s / 2\right)\right) \equiv \tau^{-1} D \hat{Q}^{2}
$$

where $v_{\mathrm{F}}=\left|\mathbf{p}_{\mathrm{F}}\right| / m$ is the magnitude of the Fermi velocity parallel to the layers. In (4), we have made the assumption $\tau D \hat{Q}^{2} \ll 1$, which we suppose throughout this manuscript and its limits have been discussed in 15 . The fluctuation propagator $L\left(\mathbf{q}, \omega_{\mu}\right)$ in the vicinity of $T_{\mathbf{c}}$ has the form :

$$
L^{-1}\left(\mathbf{q}, \omega_{\mu}\right)=-\rho\left[\epsilon+\psi\left(\frac{1}{2}+\frac{\omega_{\mu}}{4 \pi T}+\frac{4 \eta D \hat{Q}^{2}}{\pi^{2} v_{\mathrm{F}}^{2} \tau}\right)-\psi\left(\frac{1}{2}\right)\right],
$$

where $\epsilon=\ln \left(T / T_{\mathrm{c}}\right) \approx\left(T-T_{\mathrm{c}}\right) / T_{\mathrm{c}}$ for $T-T_{\mathrm{c}} \ll T_{\mathrm{c}}, \psi(x)$ is the digamma function, $\rho=N(0)=m /(2 \pi s)$ is the single-spin quasiparticle normal density of states, and

$$
\eta=-\frac{v_{\mathrm{F}}^{2} \tau^{2}}{2}\left[\psi\left(\frac{1}{2}+\frac{1}{4 \pi \tau T}\right)-\psi\left(\frac{1}{2}\right)-\frac{1}{4 \pi \tau T} \psi^{\prime}\left(\frac{1}{2}\right)\right]
$$

is the positive constant which enters into the current expression in the phenomenological GL theory in two dimensions 20 .

\section{PARACONDUCTIVITY}

Let us first examine the AL contribution (diagram 1 of Fig. 1) to the ac fluctuation conductivity. The general expression for the appropriate contribution to the electromagnetic response operator as a function of the Matsubara frequencies of the external electromagnetic field $\omega_{\nu}$ is 11 :

$$
\begin{aligned}
Q_{\alpha \beta}^{\mathrm{AL}}\left(\omega_{\nu}\right) & =2 e^{2} T \sum_{\omega_{\mu}} \int \frac{d^{3} q}{(2 \pi)^{3}} B_{\alpha}\left(\mathbf{q}, \omega_{\mu}, \omega_{\nu}\right) L\left(\mathbf{q}, \omega_{\mu}\right) \times \\
& \times L\left(\mathbf{q}, \omega_{\mu}+\omega_{\nu}\right) B_{\beta}\left(\mathbf{q}, \omega_{\mu}, \omega_{\nu}\right)
\end{aligned}
$$


The Green's functions block is defined as:

$$
\begin{aligned}
B_{\alpha}\left(\mathbf{q}, \omega_{\mu}, \omega_{\nu}\right) & =T \sum_{\omega_{n}} \int \frac{d^{3} p}{(2 \pi)^{3}} v_{\alpha}(p) C\left(\mathbf{q}, \omega_{n+\nu}, \omega_{\mu-n}\right) C\left(\mathbf{q}, \omega_{n}, \omega_{\mu-n}\right) \times \\
& \times G\left(\mathbf{p}, \omega_{n+\nu}\right) G\left(\mathbf{p}, \omega_{n}\right) G\left(\mathbf{q}-\mathbf{p}, \omega_{\mu-n}\right) .
\end{aligned}
$$

In the vicinity of $T_{\mathrm{c}}$, for frequencies $\omega \ll T$, the leading singular contribution to the response operator $Q_{\alpha \beta}^{A L(R)}$ arises from the fluctuation propagators in rather than from the frequency dependences of the $B_{\alpha}$ blocks, so it suffices to neglect its frequency dependences 17 . This approximation leads to

$$
\begin{aligned}
B_{\alpha}(\mathbf{q}, 0,0) & =-2 \rho \frac{\eta}{v_{\mathrm{F}}^{2}} \frac{\partial}{\partial q_{\alpha}}<[\xi(\mathbf{p})-\xi(\mathbf{q}-\mathbf{p})]^{2}>= \\
& =-2 \rho \frac{\eta}{v_{\mathrm{F}}^{2}} \begin{cases}s J^{2} \sin \left(q_{z} s\right) & \text { for } \alpha=z \\
v_{\mathrm{F}}^{2} q_{\alpha} & \text { for } \alpha=x, y\end{cases}
\end{aligned}
$$

Using these expressions in (8) followed by analitical continuation of the external Matsubara frequencies to the imaginary axis and integration over momenta, one can find the explicit expression for the imaginary part of the retarded electromagnetic response operatorl for real frequencies $\omega \ll T$ :

$$
\begin{gathered}
\operatorname{Im}\left[Q_{\perp}^{A L(R)}(\omega)\right]=\frac{e^{2} T}{4 \pi s}\left(\frac{s^{2}}{\eta}\right)\left(\frac{16 T_{\mathrm{c}}}{\pi \omega}\right) \operatorname{Re}\left\{\left(\frac{\pi \omega}{16 T_{\mathrm{c}}}\right)^{2}-\left(\epsilon-\frac{i \pi \omega}{16 T_{\mathrm{c}}}+\frac{r}{2}\right) \times\right. \\
\left.\times\left[\Delta D_{2}\left(\epsilon-\frac{i \pi \omega}{16 T_{\mathrm{c}}}\right)-\left(\frac{r}{2}\right)^{2} \Delta D_{1}\left(\epsilon-\frac{i \pi \omega}{16 T_{\mathrm{c}}}\right)\right]\right\} \\
\operatorname{Im}\left[Q_{\|}^{A L(R)}(\omega)\right]=\frac{2 e^{2} T}{\pi s} \operatorname{Im}\left\{\left[1+i\left(\frac{16 T_{\mathrm{c}}}{\pi \omega}\right)\left(\epsilon+\frac{r}{2}\right)\right] \times\right. \\
\left.\times\left[\Delta D_{1}\left(\epsilon-\frac{i \pi \omega}{16 T_{\mathrm{c}}}\right)\right]+i\left(\frac{16 T_{\mathrm{c}}}{\pi \omega}\right)\left[\Delta D_{2}\left(\epsilon-\frac{i \pi \omega}{16 T_{\mathrm{c}}}\right)\right]\right\}
\end{gathered}
$$

where $D_{1}(z)=2 \ln [\sqrt{z}+\sqrt{(z+r)}], D_{2}(z)=-\sqrt{z(z+r)}, \Delta D_{1}(z)=D_{1}(z)-D_{1}(\epsilon), \Delta D_{2}(z)=D_{2}(z)-D_{2}(\epsilon)$ and $r=4 \eta J^{2} / v_{\mathrm{F}}^{2}$. The value $r \sim \xi_{\mathrm{c}}^{2}(0) / s^{2}$ is the usual anisotropy parameter $\mathrm{\theta}$ characterizing the dimensional crossover from the $2 D$ to the $3 D$ regime in the thermodynamic fluctuation behavior at $T_{\mathrm{c}}$ (except for $\sigma_{\perp}$, for which the crossover is from $0 D$ to $3 D$ at $T_{\mathrm{c}}$ ). The expressions presented above solve the problem of the frequency dependence of the paraconductivity tensor in the general form for $\epsilon \ll 1$ and $\omega \lesssim T$ for an arbitrary relation between $\epsilon, r$ and $\omega$, but they are too cumbersome.

Let us concentrate on the most interesting case for the HTS analysis of $2 D$ fluctuations where $\xi_{\mathrm{c}}(T) \ll s(r \ll \epsilon)$ and $\sigma_{\perp}^{\mathrm{AL}}$ turns out to be suppressed by the necessity of the indemendent tunneling of each electron participating in the fluctuation pairing from one $\mathrm{CuO}_{2}$ layer to the neighbour one 21 . The approximation $r \ll \epsilon$ simplifies considerably the expressions (11) and (12) 3 and it keeps, in the same time, their validity up to frequencies comparable to $T_{\mathrm{c}}$ :

$$
\begin{aligned}
\sigma_{\perp}^{A L(2 D)}(\epsilon, \omega) & =\frac{e^{2} s}{64 \eta}\left(\frac{r}{2 \epsilon}\right)^{2} \frac{1}{\tilde{\omega}^{2}} \ln \left(1+\tilde{\omega}^{2}\right)= \\
& =\sigma_{\perp}^{A L(2 D)}(\epsilon, 0) \begin{cases}1-\frac{\tilde{\omega}^{2}}{2} & \text { for } \tilde{\omega} \ll 1 \\
\frac{2}{\tilde{\omega}^{2}} \ln \tilde{\omega} & \text { for } \tilde{\omega} \gg 1\end{cases}
\end{aligned}
$$

and 


$$
\begin{aligned}
\sigma_{\|}^{A L(2 D)}(\epsilon, \omega) & =\frac{e^{2}}{16 s} \frac{1}{\epsilon}\left\{\frac{2}{\tilde{\omega}} \arctan \tilde{\omega}-\frac{1}{\tilde{\omega}^{2}} \ln \left(1+\tilde{\omega}^{2}\right)\right\}= \\
& =\sigma_{\|}^{A L(2 D)}(\epsilon, 0) \begin{cases}1-\frac{\tilde{\omega}^{2}}{6} & \text { for } \tilde{\omega} \ll 1 \\
\frac{\pi}{\tilde{\omega}} & \text { for } \tilde{\omega} \gg 1\end{cases}
\end{aligned}
$$

where $\tilde{\omega}=\frac{\pi \omega}{16\left(T-T_{\mathrm{c}}\right)}$.

Let us mention two facts following from the expressions obtained. Firstly, the paraconductivity begins to decrease rapidly with the increase of frequency already for $\omega \gtrsim T-T_{\mathrm{c}}$ (the critical exponents of this power decrease coincide with those ones in $\epsilon$-dependence of dc-conductivity tensor components: $\nu_{\|}=1(2 D$ fluctuations $)$ and $\nu_{\perp}=2(0 D$ fluctuations)). Secondly the assumption to neglect the $\omega$-dependence of the Green's functions blocks evidently breaks down at frequencies $\omega \gtrsim T$ and this dependence has the only effect to accelerate the decrease.

\section{DENSITY OF STATES CONTRIBUTION}

As far as concern the DOS contribution to the electromagnetic response operator tensor the four main diagrams for it (other two of this kind are negligible in the case under consideration 11 ) are presented in Fig. 11 (2-5). The general expression for the DOS contribution to $Q_{\alpha \beta}(\omega)$ from diagram 2 is:

$$
\begin{aligned}
Q_{\alpha \beta}^{D O S(2)}\left(\omega_{\nu}\right) & =2 e^{2} T \sum_{\omega_{\mu}} \int \frac{d^{3} q}{(2 \pi)^{3}} L\left(\mathbf{q}, \omega_{\mu}\right) T \sum_{\omega_{n}} \int \frac{d^{3} p}{(2 \pi)^{3}} v_{\alpha}(\mathbf{p}) v_{\beta}(\mathbf{p}) \times \\
& \times C^{2}\left(\mathbf{q}, \omega_{n}, \omega_{\mu-n}\right) G^{2}\left(\mathbf{p}, \omega_{n}\right) G\left(\mathbf{q}-\mathbf{p}, \omega_{\mu-n}\right) G\left(\mathbf{p}, \omega_{n+\nu}\right),
\end{aligned}
$$

The external frequency $\omega_{\nu}$ enters the expression (15) only by the Green's function $G\left(\mathbf{p}, \omega_{n+\nu}\right)$ and it is not involved in $\mathbf{q}$ integration. So, near $T_{\mathrm{c}}$, even in the case of an arbitrary external frequency, we can choose the propagator frequency $\omega_{\mu}=0$. In the same way it can be treated the contribution from diagram 3 of Fig. 11.

The diagrams 2,4 and 3,5 of Fig. 1 are topologically equivalent and this fact would let think that they give equal contributions to $\sigma(\omega)$. Nevertheless the thoroughful analysis of the analitical continuation over the external frequency show 1 that their contributions differ slightly and for the total DOS contribution to conductivity one can find:

$$
\operatorname{Re}\left(\begin{array}{c}
\sigma_{\perp}^{\mathrm{DOS}}(\omega) \\
\sigma_{\|}^{\mathrm{DOS}}(\omega)
\end{array}\right)=-\frac{e^{2}}{2 \pi s}\left(\begin{array}{c}
\frac{s^{2} J^{2}}{v_{\mathrm{F}}^{2}} \\
1
\end{array}\right) \ln \left[\frac{2}{\sqrt{\epsilon+r}+\sqrt{\epsilon}}\right] \kappa(\omega, T, \tau)
$$

where

$$
\begin{aligned}
& \kappa(\omega, T, \tau)=\frac{T v_{\mathrm{F}}^{2}}{\eta} \frac{1}{\left(\tau^{-2}+\omega^{2}\right)^{2}}\left\{\frac{4}{\tau}\left[\psi\left(\frac{1}{2}\right)-\operatorname{Re} \psi\left(\frac{1}{2}-\frac{i \omega}{2 \pi T}\right)\right]+\frac{\tau^{-2}+\omega^{2}}{4 \pi T \tau} \frac{1}{\omega} \operatorname{Im} \psi^{\prime}\left(\frac{1}{2}-\frac{i \omega}{2 \pi T}\right)+\right. \\
& \left.+\left(\tau^{-2}-\omega^{2}\right) \frac{1}{\omega}\left[\operatorname{Im} \psi\left(\frac{1}{2}-\frac{i \omega}{2 \pi T}\right)-2 \operatorname{Im} \psi\left(\frac{1}{2}-\frac{i \omega}{4 \pi T}+\frac{1}{4 \pi T \tau}\right)\right]\right\} .
\end{aligned}
$$

Let us stress that, in contrast to (11) and (12), this result has been found with the only assumption $\epsilon \ll 1$, so it is valid for any frequency, any impurity concentration and any dimensionality of the fluctuation behavior. The function $\kappa(\omega, T, \tau)$ can be easily used to fit experimental data. Nevertheless we present the asymptotics of the expression (17) for clean and dirty cases. In the dirty case

$$
\kappa_{\mathrm{d}}\left(\omega, T \ll \tau^{-1}\right)=\frac{T v_{\mathrm{F}}^{2}}{2 \eta} \begin{cases}\frac{\tau}{(2 \pi T)^{2}}\left|\psi^{\prime \prime}\left(\frac{1}{2}\right)\right| & \text { for } \omega \ll T \ll \tau^{-1} \\ \frac{\tau}{\omega^{2}} & \text { for } T \ll \omega \ll \tau^{-1} \\ -\frac{\pi}{\omega^{3}} & \text { for } T \ll \tau^{-1} \ll \omega\end{cases}
$$


and in the clean case

$$
\kappa_{\mathrm{cl}}\left(\omega, T \gg \tau^{-1}\right)=\frac{T v_{\mathrm{F}}^{2}}{2 \eta} \begin{cases}\frac{\pi \tau^{2}}{4 T} & \text { for } \omega \ll \tau^{-1} \ll T \\ -\frac{\pi}{4 \omega^{2} T} & \text { for } \tau^{-1} \ll \omega \ll T \\ -\frac{\pi}{\omega^{3}} & \text { for } \tau^{-1} \ll T \ll \omega\end{cases}
$$

\section{MAKI-THOMPSON CONTRIBUTION}

The total contribution of the MT-like diagrams to $\sigma_{\alpha \beta}(\omega)$ has been analysed in 11 in the case of zero frequency and the frequency dependence of $\sigma_{\|}(\omega)$ has been studied in 17 . In 11 it was shown that actually the regular part of MT diagram can almost always be omitted. So we will not discuss it in this paper and we will concentrate on the anomqlous MT contribution (Fig. 1 diagram 6).

In 17 it was demonstrated that in the case of quasi two-dimensional electron motion (2) there is no formal necessity to introduce the pairbreaking time $\tau_{\varphi}$ because the Maki-Thompson logarithmic divergency is automatically cut off due to the possible interlayer hopping. Nevertheless, all evidences show that the intrinsic pairbreaking in HTS is strong (at least one of its sources may be identified as thermal phonons) and an estimation of the appropriate $\tau_{\varphi} \sim 2 \div 5 \cdot 10^{-13} s$ is only several times larger than $T_{c}^{-1}$. So we have to speak actually about the overdamped regime for Maki-Thompson contribution and it doesn't manifest itself noticeably in the $\epsilon$ dependence of conductivity 11 (the major part of experimental results is explained in terms of AL or AL and DOS contributions). Anyway we are interested in MT contribution because of its frequency dependence which evidently determines another characteristic scale in addition to the previous three $\left(T-T_{\mathrm{c}}, T, \tau^{-1}\right)$ we have introduced: $\omega_{\mathrm{MT}} \sim \tau_{\varphi}^{-1}$.

We start, as usual, from the general expression of the anomalous MT contribution to the electromagnetic operator tensor 11 :

$$
Q_{\alpha \beta}^{\mathrm{MT}}\left(\omega_{\nu}\right)=2 e^{2} T \sum_{\omega_{\mu}} \int \frac{d^{3} q}{(2 \pi)^{3}} L\left(\mathbf{q}, \omega_{\mu}\right) I_{\alpha \beta}\left(\mathbf{q}, \omega_{\mu}, \omega_{\nu}\right),
$$

where

$$
\begin{aligned}
I_{\alpha \beta}\left(\mathbf{q}, \omega_{\mu}, \omega_{\nu}\right) & =T \sum_{\omega_{n}} \int \frac{d^{3} p}{(2 \pi)^{3}} v_{\alpha}(\mathbf{p}) v_{\beta}(\mathbf{q}-\mathbf{p}) C\left(\mathbf{q}, \omega_{n+\nu}, \omega_{\mu-n-\nu}\right) C\left(\mathbf{q}, \omega_{n}, \omega_{\mu-n}\right) \times \\
& \times G\left(\mathbf{p}, \omega_{n+\nu}\right) G\left(\mathbf{p}, \omega_{n}\right) G\left(\mathbf{q}-\mathbf{p}, \omega_{\mu-n-\nu}\right) G\left(\mathbf{q}-\mathbf{p}, \omega_{\mu-n}\right) .
\end{aligned}
$$

After the integration over $\mathbf{p}$ momentum and the summation over $\omega_{n}$ in the range $\omega_{n} \in\left[-\omega_{\nu}, 0[\right.$ (anomalous part), one can find:

$$
\begin{aligned}
& \left(\begin{array}{l}
Q_{\perp}^{\mathrm{MT}(\mathrm{an})}\left(\omega_{\nu}\right) \\
Q_{\|}^{\mathrm{MT}(\mathrm{an})}\left(\omega_{\nu}\right)
\end{array}\right)=e^{2} T \tau\left[\psi\left(\frac{1}{2}+\frac{\omega_{\nu}}{2 \pi T}\right)-\psi\left(\frac{1}{2}\right)\right] \times \\
& \times \int \frac{d^{3} q}{(2 \pi)^{3}}\left(\begin{array}{c}
J^{2} s^{2} \cos q_{\perp} s \\
v_{\mathrm{F}}^{2}
\end{array}\right) \frac{1}{\left(\omega_{\nu}+\tau_{\varphi}^{-1}+D \hat{Q}^{2}\right)\left(\epsilon+\eta q^{2}+r \sin ^{2}\left(q_{\perp} s / 2\right)\right)}
\end{aligned}
$$

At this stage of calculations we artificially introduce the phase-breaking time in the "Cooperon" vertices. Carrying out the integration and separating the real and the imaginary parts we have:

$$
\begin{aligned}
& \operatorname{Re}\left(\begin{array}{c}
\sigma_{\perp}^{\mathrm{MT}(\mathrm{an})}(\omega) \\
\sigma_{\|}^{\mathrm{MT}(\mathrm{an})}(\omega)
\end{array}\right)=\frac{e^{2}}{2 \pi s}\left(\begin{array}{c}
s^{2} \\
2 \eta \\
1
\end{array}\right) \frac{T}{\omega} \operatorname{Im}\left\{\frac{\psi\left(\frac{1}{2}-\frac{i \omega}{2 \pi T}\right)-\psi\left(\frac{1}{2}\right)}{\frac{i \pi \omega}{8 T_{\mathrm{c}}}+\epsilon-\gamma} \times\right. \\
& \left.\times\left(\begin{array}{c}
-\Delta D_{2}\left(-\frac{i \pi \omega}{8 T_{\mathrm{c}}}+\gamma\right)+\left(\frac{i \pi \omega}{8 T_{\mathrm{c}}}+\epsilon-\gamma\right) \\
\Delta D_{1}\left(-\frac{i \pi \omega}{8 T_{\mathrm{c}}}+\gamma\right)
\end{array}\right)\right\}
\end{aligned}
$$


where $\gamma=\frac{\pi}{8 T_{\mathrm{c}} \tau_{\varphi}}$. In the case of two-dimensional overdamped regime $(r \ll \epsilon \lesssim \gamma)$ the expression (23) gives the following limits:

$$
\begin{gathered}
\sigma_{\perp}^{M T(a n)(2 D)}(\omega)=\frac{e^{2} s}{2^{7} \eta} \frac{r^{2}}{\gamma \epsilon} \begin{cases}1 & \text { for } \omega \ll \tau_{\varphi}^{-1} \\
\left(\frac{8 T_{\mathrm{c}} \gamma}{\pi \omega}\right)^{2} & \text { for } \omega \gg \tau_{\varphi}^{-1}\end{cases} \\
\sigma_{\|}^{M T(a n)(2 D)}(\omega)=\frac{e^{2}}{8 s} \begin{cases}\frac{1}{\gamma} \ln \left(\frac{\gamma}{\epsilon}\right) & \text { for } \omega \ll \tau_{\varphi}^{-1} \\
\frac{4 T_{\mathrm{c}}}{\omega} & \text { for } \omega \gg \tau_{\varphi}^{-1}\end{cases}
\end{gathered}
$$

Let us remind that the expression (23) has been obtained without any limitation on frequency. Nevertheless we have done the assumption $\tau D \hat{Q}^{2} \ll 1$ through all the paper and it turns out that, while this condition doesn't restrict our results for the AL and the DOS contributions over the full range of frequency, temperature and impurity concentration, this is not so for the MT contribution. In fact, as it was shown in 15 , in the ultra-clean (or non-local) limit, when $T \tau>1 / \sqrt{\epsilon}$, the assumption $\tau D \hat{Q}^{2} \ll 1$ is violated for MT contribution and the results obtained are not valid there. Nevertheless one can see that this non-local situation can be realized in the clean case $(T \tau \gg 1)$ only and for temperatures in the range $1 /(T \tau)^{2} \ll \epsilon \ll 1$. We suppose $T \tau \sim 1$, as it is in the case of HTS, so we skip the discussion about the non-local limit.

\section{DISCUSSION}

Let us start from the analysis of each fluctuation contribution separately and then we will discuss their interplay in $\operatorname{Re}\left[\sigma_{\perp}(\omega)\right]$. Because of the large number of parameters entering the expressions we restrict our consideration to the $c$-axis component of the conductivity tensor in the region of $2 D$ fluctuations (above Lawrence-Doniach crossover temperature). The in-plane component will be overviewed in the end of this section.

The AL contribution describes the fluctuation condensate response to the electromagnetic field applied. The component of the current associated with it can be treated as the precursor phenomenon of the screening currents in the superconducting phase. Above $T_{\mathrm{c}}$ the virtual Cooper pairs binding energy gives rise to a pseudogap of the order of $T-T_{\mathrm{c}}$, so it is not surprising that at higher frequencies the AL contribution decreases with the further increase of $\omega$. Actually $\omega^{\mathrm{AL}} \sim T-T_{\mathrm{c}}$ is the only relevant scale for $\sigma^{\mathrm{AL}}$ : its frequency dependence doesn't contain $T, \tau_{\phi}$ and $\tau$. The independence from the latter manifests mathematically the fact that elastic impurities do not represent obstacles for the motion of Cooper pairs. The interaction of the electromagnetic wave with the fluctuation Cooper pairs resembles, in some way, the anomalous skin-effect where its reflection is determined by the interaction with the free electron system.

Another effect related with the formation of the fluctuation Cooper pairs, but on the self-intersecting trajectories (like the weak localization correction), is described by the MT anomalous contribution. Being the contribution related with the Cooper pairs electric charge transfer it doesn't depend on the elastic scattering time but it turns out to be extremely sensitive to the phase-breaking mechanisms. So two characteristic scales turn out to be relevant in the frequency dependence of that one: $T-T_{\mathrm{c}}$ and $\tau_{\phi}^{-1}$. In the case of HTS, where $\tau_{\phi}^{-1}$ has to be estimated as at least $0.1 T_{\mathrm{c}}$ for temperatures up to $5 \div 10 \mathrm{~K}$ above $T_{\mathrm{c}}$, the MT contribution is overdamped, it is determined by the value of $\tau_{\phi}$ and it almost does not depend on temperature.

The density of states fluctuation renormalization gives quite different contribution to $\operatorname{Re}[\sigma(\omega)]$ with respect to those above. Physically it is related with the decrease of the one electron density due to the involvement of some number of electrons in the fluctuation Cooper pairing. At low frequencies $\left(\omega \ll \tau^{-1}\right)$ the lack of electron states on the Fermi level leads to an opposite effect in comparison with AL and MT contributions: $\operatorname{Re}\left[\sigma^{\mathrm{DOS}}(\omega)\right]$ turns out to be negative and this means the increase of the surface impedance, or, in other words, the decrease of reflectance. Nevertheless, the electromagnetic field applied affects the electron distribution and at high frequencies $\omega \sim \tau^{-1}$ the DOS contribution changes its sign. It is interesting that DOS contribution, as one-electron effect, depends on the impurity scattering similiarly to the normal Drude conductivity. The decrease of $\operatorname{Re}\left[\sigma^{\mathrm{DOS}}(\omega)\right]$ starts already at frequencies $\omega \sim \min \left\{T, \tau^{-1}\right\}$ which for HTS are much higher than $T-T_{\mathrm{c}}$ and $\tau_{\varphi}^{-1}$.

The scenario of $\operatorname{Re}\left[\sigma_{\perp}^{\text {tot }}\right] \omega$-dependence with the most natural choice of parameters $\left(r \ll \epsilon \lesssim \tau_{\varphi}^{-1} \ll \min \left\{T, \tau^{-1}\right\}\right)$ is presented in Fig. 2. The positive AL and MT effects, in their $\omega$-dependence, are well pronounced at low frequencies on the background of the DOS contribution which remains in this region a negative constant. Then at $\omega \sim T-T_{\mathrm{c}}$ 
the former decays and the $\operatorname{Re} \sigma_{\perp}$ remains negative up to $\omega \sim \min \left\{T, \tau^{-1}\right\}$. The DOS correction changes its sign at $\omega \sim \tau^{-1}$ and then it rapidly decreases. The following high frequency behavior is governed by the Drude law. So one can see that the characteristic pseudogap-like behavior in the frequency dependence of the optical conductivity, mentioned in the title of this paper, takes place in the range $\omega \in\left[T-T_{\mathrm{c}}, \tau^{-1}\right]$. The depth of the window increases logarithmically with $\epsilon$ when $T$ tends to $T_{\mathrm{c}}$, as shown in Fig. 3. In the case of $a b$-plane optical conductivity the two first positive contributions are not suppressed by the interlayer transmittance, they exceed considerably the negative DOS contribution in a wide range of frequencies and any pseudogap-like behavior is unlikely in $\sigma_{\|}^{\mathrm{fl}}(\omega)$ : the reflectivity has to be of the metal kind. The comparison between (14) and (16) shows that the compensation of the two contributions could only take place at $\omega_{0} \sim T / \ln \epsilon$ which is out of the range of validity of the AL contribution.

Let us compare now the results of our calculations with the experiments available. The recent measurements 22 , 23 of the $c$-axis reflectivity spectra, in the FIR region on $\mathrm{YBa}_{2} \mathrm{Cu}_{4} \mathrm{O}_{8}$ single crystals, show the response of a poor metal with the additional contributions from $I R$ active phonon modes (which we do not discuss here). With the decrease of temperature the $c$-axis optical conductivity decreases showing a transition from a Drude-like to a pseudogap-like behavior at $\omega \sim 180 \mathrm{~cm}^{-1}$. This gap grows deeper below $180 \mathrm{~K}$ without any abrupt change at the superconducting transition temperature $T_{\mathrm{c}}=80 \mathrm{~K}$.

Such experimentally observed behavior of the optical conductivity is in qualitative agreement with our results. Really the suppression of the density of states due to the superconducting fluctuations in the vicinity of $T_{\mathrm{c}}$ leads to the decrease of reflectivity in the range of frequencies up to $\omega \sim \tau^{-1}$. The magnitude of this depression slowly (logarithmically) increases with the decrease of temperature but, evidently, at the edge of the transition it reaches some saturation because of the crossover to the $3 D$ regime in fluctuations (where instead of $\ln (1 / \epsilon)$ one has $\ln (1 / r)-\sqrt{\epsilon}$, see (16)). So no singularity is expected in the value of the minimum even in the first order of perturbation theory. Below $T_{\mathrm{c}}$ the fluctuation behavior of $\left\langle\Delta_{\mathrm{fl}}^{2}\right\rangle$ is mostly symmetrical to that one above $T_{\mathrm{c}}$ (see 24 ) and, with the further decrease of temperature, the fluctuation pseudogap minimum in the optical conductivity smoothly transforms itself into the real superconducting gap, which opens, in HTS, very sharply. Let us stress, that the independence of the pseudogap threshold from temperature appears naturally in our theory: it is determined by $\omega_{0} \sim \tau^{-1}$ (see (18), (19) and Fig. (1). Comparing Fig. 3 e Fig. 4, one can easy see that the threshold doesn't move when the temperature changes but varies when the inverse of the scattering rate changes. As far as concern the numerical value of $\omega_{0}$, supposing $T_{\mathrm{c}} \tau=0.35$ (which is the value for the scattering rate of the sample used in the experiment under consideration 23 , that is also in the experimental range of the inverse of the scattering rate $T \tau \approx 0.3 \div 0.225 .26$ ), one can see that the pseudogap threshold is of the order of $200 \mathrm{~cm}^{-1}$, in agreement with the experimental data, even from a quantitative point of view22 23 .

Our theory is, strictly speaking, valid only in the vicinity of the critical temperature, where $\epsilon \ll 1$. Nevertheless the logarithmic dependence on $\epsilon$ of the result obatained gives grounds to believe that qualititatively the theory can be valid up to $\epsilon=\ln \left(T / T_{\mathrm{c}}\right) \sim 1$, so for temperatures up to $200 \mathrm{~K}$ in the experiment discussed. So the theory is again in agreement with the experimental value of temperature $180 \mathrm{~K}$ until which the pseudogap is observable.

In conclusion we have calculated the tensor of optical conductivity for layered superconductors. The pseudogap-like minimum of its $c$-axis component in a wide range of frequencies for temperatures in the vicinity of $T_{\mathrm{c}}$ is found. Its origin is related with the fluctuation density of states renormalization which can be treated as the opening of the fluctuation pseudogap. These result are qualitatively, and in some aspects quantitatively, in agreement with the recent experiments on $\mathrm{YBa}_{2} \mathrm{Cu}_{4} \mathrm{O}_{8}$ samples. Further experiments, with more anisotropic samples like $\mathrm{BSSCO}$ single crystals, would be useful, because the effect should be more prononced.

\section{ACKNOWLEDGMENTS}

We would like to thank P. Calvani, M. Capizzi, C. Di Castro, D. Livanov, M. Randeria and V. Tognetti for valuable discussions. This work was partially supported by NATO Collaborative Research Grant \#CRG941187. 
${ }^{1}$ On leave of absence from Department of Theoretical Physics, Moscow Institute of Steel and Alloys.

${ }^{2}$ It is necessary to mention that the direct falculation of the expression (8) leads to the appearence of divergent expressions in the $\operatorname{Re} Q$. Nevertheless, as it was shown in 17 , the thorough summation of all diagrams from the first order of the perturbation theory for $Q$ before momentum integration leads to the exact cancellation of $\operatorname{Re}\left[Q^{\mathrm{f}}(0)\right]$. Namely this fact justifies the possibility of the further calculation of different diagrammatifiterms to $Q^{\mathrm{fl}}$ separately.

3 The second expression coincides with that one obtained in 17.18

${ }^{4}$ This fact had not been taken into account before and it led to the lack of the factor $1 / 2$ in the dirty and clean limit of the DOS contribution 511. We want to thank D. Livanov who attracted our attention to this circumstance.

${ }^{5}$ L. B. Ioffe, A. I. Larkin, A. A. Varlamov, L. Yu, Phys. Rev. B 47, 8936(1993).

${ }^{6}$ K. E. Gray, D. H. Kim, Phys. Rev. Lett. 70, 1693 (1993).

${ }^{7}$ G. Balestrino, M. Marinelli, E. Milani, L. Yu, Phys. Rev. B 47, 6037 (1993).

${ }^{8}$ E. Abrahams, M. Redi, J.W. Woo, Phys. Rev. B 1, 208 (1970).

${ }^{9}$ C. Di Castro, C. Castellani, R. Raimondi, A. Varlamov, Phys. Rev. B 49,10211 (1990).

${ }^{10}$ G. Balestrino, E. Milani, A. Varlamov, Physica C 210, 386 (1993).

${ }^{11}$ V. V. Dorin, R. A. Klemm, A. A. Varlamov, A. I. Buzdin, D. V. Livanov, Phys. Rev. B 48, 12951 (1993).

12 G. Balestrino, E. Milani, and A. A. Varlamov Soviet JETP Letters 61, 833 (1995).

13 S. Alexander, T. Maniv, Physica C 9, 1699 (1976).

${ }^{14}$ K. Kuboki, H. Fukuyama, J. Phys. Soc. Jpn 58, 376 (1989).

15 M. Randeria and A. A. Varlamov, Phys. Rev. B 50, 10401 (1994).

${ }^{16}$ P. Carretta, D. Livanov, A. Rigamonti, A. A. Varlamov Proceedings of the General Conference of the European Physics Society, Stresa, Italy, (1996)

${ }^{17}$ L. G. Aslamazov and A. A. Varlamov, Journ. of Low Temp. Phys. 38, 223 (1980).

18 H. Schmidt, Ann. Phys. Lpz 216, 336 (1968)

19 B. L. Al'tshuler, M. Yu. Reyzer, A. A. Varlamov, JETP 57, 1329 (1983).

${ }^{20}$ L.P.Gor'kov, Sov. JETP 9, 1364 (1959).

${ }^{21}$ L. B. Ioffe, A. I. Larkin, A. A. Varlamov, L. Yu, Physica C 235, 1963 (1994).

${ }^{22}$ D. N. Basov, T. Timusk, B. Dabrowski, H. A. Mook, Phys. Rev B 52, 13141 (1995).

${ }^{23}$ D. N. Basov, T. Timusk, B. Dabrowski and J. D. Jorgensen, Phys. Rev. B 50, 3511 (1994).

${ }^{24}$ A. A. Varlamov, V. V. Dorin, Sov. JETP 64, 1159 (1986); A. A. Varlamov, V. V. Dorin, I. E. Smolyarenko, Sov. JETP 67, 2536 (1988).

${ }^{25}$ D. B. Tanner and T. Timusk, "Optical Properties of High $T_{\mathrm{c}}$ Superconductors" in Properties of High Temperature Superconductors III, edited by D. M. Ginsberg, World Scientific Singapore, 428 (1992).

${ }^{26}$ P. Calvani, "Infrared Optical Properties of High- $T_{\mathrm{c}}$ Cuprates" in High $T_{\mathrm{c}}$ Superconductivity: Theory and Experiments, edited by M. Acquarone, World Pub. Co., Singapore, 233 (1996). 
FIG. 1. We report the leading Feynman diagrams contributing to the optical conductivity. The wavy lines indicate the fluctuation propagators; the thin solid lines with arrows stand for the impurity-averaged normal-state electron Green's functions; the shaded partial circles are the vertex corrections arising from impurities; the dashed curves with central crosses are additional impurity renormalizations. Diagram 1 is the Aslamazov-Larkin contribution, diagrams 2-5 are the corrections from the density of states renormalization and diagram 6 is the Maki-Thompson diagram. 
FIG. 2. The plot shows the dependence of the real part of conductivity, normalized on the Drude normal conductivity, on $\omega / T, \Re\left[\sigma^{\prime}(\omega)\right]=\operatorname{Re}[\sigma(\omega)] / \sigma^{\mathrm{n}}$. The dashed line refers to the $a b$-plane component of the conductivity tensor whose Drude normal conductivity is $\sigma_{\|}^{\mathrm{n}}=\rho e^{2} \tau v_{\mathrm{F}}^{2}$. The solid line refers to the $c$-axis component whose Drude normal conductivity is $\sigma_{\perp}^{\mathrm{n}}=\sigma_{\|}^{\mathrm{n}} J^{2} s^{2} / v_{\mathrm{F}}^{2}$. In this plot we have put $T \tau=0.3, E_{\mathrm{F}} / T=50, r=0.01, \epsilon=0.04, T \tau_{\varphi}=4$. 
FIG. 3. The behavior of the $c$-axis component of conductivity frequency dependence, for different values of temperature, is shown. The solid line refers to $\epsilon=0.04$; the dashed line refers to $\epsilon=0.06$; the dot-dashed line refers to $\epsilon=0.08$. $T \tau=0.2$ for all the curves. The other parameters of this plot are the same used in Fig. 2. 
FIG. 4. The plot shows the dependence of $\operatorname{Re}\left[\sigma_{\perp}(\omega) / \sigma_{\perp}^{\mathrm{n}}\right]$ on $\omega / T$ for different values of $T \tau$. The solid line refers to $T \tau=0.4$; the dot-dashed line refers to $T \tau=0.3$; the dashed line refers to $T \tau=0.2$. The other parameters of this plot are the same used in Fig. 目. 